\title{
Working with vulnerable families in deprived areas
}

Working with vulnerable families is an everyday aspect of general practice in severely deprived areas. Through many types of contact, practice teams acquire substantial knowledge about the most vulnerable families in their registered populations. Their frustration is not being able to help more effectively at an early stage.

Key issues are how this knowledge is acquired, how it should be used and how to link with other professions and services. Several recent NHS developments have undermined the knowledge that practice teams acquire. For example, the withdrawal of child surveillance in deprived areas is considered a mistake, given the high yield of health and social problems. Burnout and loss of staff due to excessive caseloads removes from practices the knowledge, experience, and relationships that colleagues have developed over many years.

Pregnancy is an important opportunity to demonstrate how professions and services can work together. No one argues that midwives are best placed to lead on matters directly concerned with the pregnancies and deliveries of most women but the main causes of maternal mortality and morbidity during pregnancy are no longer obstetric in nature. A review of the issues identified at booking in one severely deprived general practice population ${ }^{1}$ shows the wide range of associated problems including addiction, mental health, ethnicity, and language, in addition to medical problems. For such women, care during pregnancy should not be an isolated episode but part of a continuing, coordinated process. General practice can add the flexibility, unconditionality, accessibility (often 'the last stop', when other services do not respond) and continuity that families need.

At a meeting ${ }^{1}$ on working with vulnerable children and families, attended by 20 Deep End GPs and 60 colleagues from other professions and services, two GPs gave powerful presentations on their ability to deliver coordinated, personal continuity of care for pregnant women with complex problems. Unusually, one presentation was able to describe the long-term benefits of a practice-attached midwife, while the other described the advantages of working with a practice-attached social worker. Whether working with patients or with professional colleagues, the active ingredient is a longterm relationship based on communication, mutuality, and trust.

All agreed that current resource provision is inadequate to stem the tide of vulnerable families which recent economic and social policies in the UK have helped to produce. There was also concern that by concentrating scarce professional resources, such as health visitors, on the highest risk families, less preventive work will be done to reduce the number of families progressing to high-risk status. The policy may be counter-productive before long.

When time is short and caseloads are large, thresholds for intervention are bound to rise. Practice teams are often aware of vulnerable children and families before serious problems develop, but lack the resources to intervene. Investments are needed in home support, free nursery places and other ways of supporting families.

To address the inverse care law, the GP contract and/or enhanced service agreements should explicitly support practices in working with vulnerable families in ways that are commensurate with the numbers of vulnerable families within practices, and not just the numbers of cases on child protection registers. Practices should identify their lead professional for vulnerable families, coordinating activities within the practice and considering the ways in which they could work more effectively with other practices and with other professions and services, including social workers and school nurses.

Effective joint working depends on colleagues knowing each other's names,

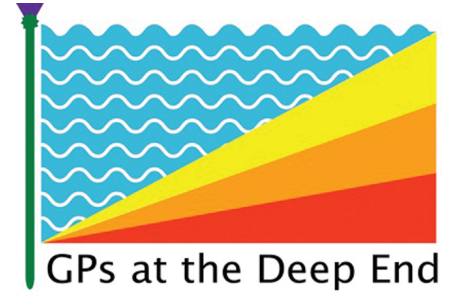

being well informed about each other's roles, how they may be contacted locally and the constraints under which they work. Professionals and services should be accountable not only for their own contribution but also how this connects with the contributions of others. The 'connectedness' of care should be a major policy, management, and practitioner objective. The hallmarks of a caring system are not only the quality of encounters between practitioners and families, but also the extent to which the system measures itself in providing needs-based support to all who need it, matches rhetoric about joint working by measures to support and review joint working, provides continuity of care, and assesses itself against a range of outcomes, including the views of parents and children. A caring system should also care for its staff, ensuring reasonable caseloads, sharing the burden and finding practical ways of encouraging and rewarding commitment and continuity.

The Deep End meeting provided an example of how practitioners and managers from different services can learn from each other, share experiences, correct misperceptions, and discuss how services can be improved. The extraordinary nature of the meeting needs to be made ordinary, as part of a learning organisation, dedicated to supporting professionals and services working with vulnerable children and families.

\section{Graham Watt}

On behalf of the Deep End Steering Group. This is the fourth article in a series on General Practitioners at the Deep End.

\section{REFERENCE}

1. Deep End Report 12. Working together for vulnerable children and families. The twelfth meeting of 'General Practitioners at the Deep End', 9 September 2010. http://www.gla.ac.uk/media/media_183114_en.pdf (accessed 9 Mar 2011).

DOI: 10.3399/bjgp11X567306 